\title{
THE C+N+O ABUNDANCE OF $\omega$ CENTAURI GIANT STARS: IMPLICATIONS FOR THE CHEMICAL-ENRICHMENT SCENARIO AND THE RELATIVE AGES OF DIFFERENT STELLAR POPULATIONS*
}

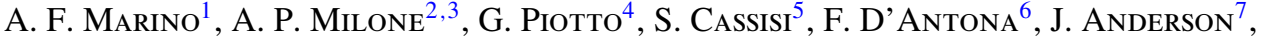 \\ A. Aparicio ${ }^{2,3}$, L. R. Bedin ${ }^{8}$, A. Renzini ${ }^{8}$, And S. Villanova $^{9}$ \\ ${ }^{1}$ Max-Planck-Institut für Astrophysik, Karl-Schwarzschild-Str. 1, 85741 Garching bei München, Germany; amarino@ MPA-Garching.MPG.DE \\ ${ }^{2}$ Instituto de Astrofísica de Canarias, E-38200 La Laguna, Tenerife, Canary Islands, Spain; milone@iac.es, aparicio@iac.es \\ ${ }^{3}$ Department of Astrophysics, University of La Laguna, E-38200 La Laguna, Tenerife, Canary Islands, Spain \\ ${ }^{4}$ Dipartimento di Astronomia, Università di Padova, Vicolo dell’Osservatorio 3, Padova, I-35122, Italy; giampaolo.piotto@ unipd.it \\ ${ }^{5}$ INAF-Osservatorio Astronomico di Teramo, Via M. Maggini, 64100 Teramo, Italy; cassisi@ oa-teramo.inaf.it \\ ${ }^{6}$ INAF-Osservatorio Astronomico di Roma, via Frascati 33, I-00040 Monteporzio, Italy; dantona @ oa-roma.inaf.it \\ ${ }^{7}$ Space Telescope Science Institute, 3700 San Martin Drive, Baltimore, MD 21218, USA; jayander@ stsci.edu \\ ${ }^{8}$ INAF-Osservatorio Astronomico di Padova, Vicolo dellOsservatorio 5, 35122 Padova, Italy; luigi.bedin@ oapd.inaf.it, alvio.renzini@oapd.inaf.it \\ ${ }^{9}$ Departamento de Astronomía, Universidad de Concepción, Casilla 160-C, Concepción, Chile; svillanova@ @astro-udec.cl \\ Received 2011 June 30; accepted 2011 November 2; published 2012 January 19
}

\begin{abstract}
We present a chemical-composition analysis of 77 red-giant stars in Omega Centauri. We have measured abundances for carbon and nitrogen, and combined our results with abundances of $\mathrm{O}, \mathrm{Na}, \mathrm{La}$, and $\mathrm{Fe}$ that we determined in our previous work. Our aim is to better understand the peculiar chemical-enrichment history of this cluster by studying how the total $\mathrm{C}+\mathrm{N}+\mathrm{O}$ content varies among the different metallicity stellar groups, and among stars at different places along the $\mathrm{Na}-\mathrm{O}$ anticorrelation. We find that the (anti)correlations among the light elements that would be expected on theoretical grounds for matter that has been nuclearly processed via high-temperature proton captures. The overall $[(\mathrm{C}+\mathrm{N}+\mathrm{O}) / \mathrm{Fe}]$ increases by $\sim 0.5$ dex from $[\mathrm{Fe} / \mathrm{H}] \sim-2.0$ to $[\mathrm{Fe} / \mathrm{H}] \sim-0.9$. Our results provide insight into the chemical-enrichment history of the cluster, and the measured $\mathrm{CNO}$ variations provide important corrections for estimating the relative ages of the different stellar populations.
\end{abstract}

Key words: globular clusters: individual (NGC 5139) - stars: abundances - stars: Population II

Online-only material: color figures

\section{INTRODUCTION}

Omega Centauri ( $\omega$ Cen) is one of the most intriguing globular clusters (GCs) of the Galaxy. At odds with the majority of GCs, which are mono-metallic, it shows large star-to-star metallicity variations up to more than 1 dex (e.g., Norris et al. 1996; Suntzeff $\&$ Kraft 1996). At the same time, it shares with the most monometallic GCs the presence of a $\mathrm{Na}-\mathrm{O}$ anticorrelation, which is present across almost the entire metallicity range (Marino et al. 2011a; Johnson \& Pilachowski 2010). These two facts suggest unique complexity in the cluster's star formation history and its chemical evolution.

The complexity of $\omega$ Cen also manifests itself in its color-magnitude diagram (CMD) with the presence of multiple red-giant branches (RGBs), multiple sub-giant branches (SGBs; Lee et al. 1999; Pancino et al. 2000), and an extended horizontal branch (Villanova et al. 2007; Cassisi et al. 2009; D'Antona et al. 2010; Bellini et al. 2010). The main sequence (MS) is double (Anderson 1998), and a third, less-populated MS has been discovered by Bedin et al. (2004) and associated with the most metal-rich population. The bluer MS (bMS) has a higher metallicity than the red MS (rMS; Piotto et al. 2005). So far, the only way to account for these observations is to assume the bMS to be highly enhanced in He (Norris 2004; Bedin et al. 2004; Piotto et al. 2005). Direct evidence in these directions has been recently detected from the analysis of the He I 10830 transition on $\omega$ Cen RGB stars (Dupree et al. 2011). The correlation

\footnotetext{
* Based on data collected at the European Southern Observatory with the FLAMES/GIRAFFE spectrograph.
}

of the $\mathrm{He}$ line detection with $[\mathrm{Fe} / \mathrm{H}], \mathrm{Al}$, and $\mathrm{Na}$ supports the assumption that helium is enhanced in the bMS.

Due to the observational scenario, being more complex than in any other GC, it has often been suggested (e.g., Bekki \& Freeman 2003) that $\omega$ Cen may be the remnant of a nowdissolved dwarf galaxy, once similar to the Sagittarius dwarf (with its central GC M54) now being torn apart by the Galactic tidal field. In any case, a successful description of the $\omega$ Cen star formation history should be able to explain both the $\mathrm{Na}-\mathrm{O}$ anticorrelation at the various metallicities and the general rise in slow-process (s-process) elements as a function of metallicity (Norris \& Da Costa 1995; Johnson \& Pilachowski 2010; Smith et al. 2000; Marino et al. 2011a). The presence of the $\mathrm{Na}-\mathrm{O}$ anticorrelation implies that $\omega$ Cen, similar to mono-metallic $\mathrm{GCs}$, has experienced enrichment from high-temperature H-burning processed material. At the same time, an additional physical mechanism should be present to produce $s$-process elements. In the Sun, the s-process elements' abundance is mainly due to two components: the main component (attributed to lowmass AGB stars of 1.5-3 $M_{\odot}$; Busso et al. 1999) and the weak component (attributed to massive stars; see Raiteri et al. 1993 and references therein). At solar metallicity, this last component mainly produces the lighter $s$-process nuclei, at most up to $\mathrm{Sr}$ and $\mathrm{Kr}$ (Raiteri et al. 1993). In the case of $\omega$ Cen, if we assume that the $s$-elements are produced in the strong component, i.e., in a population of less massive asymptotic-giant-branch (AGB) stars (Busso et al. 1999; Ventura et al. 2009), we have to face a timescale discrepancy that makes it hard to solve the entire puzzle (D'Antona et al. 2011). Indeed, these AGBs have longer lifetimes than the more massive AGB stars, which are presumed 


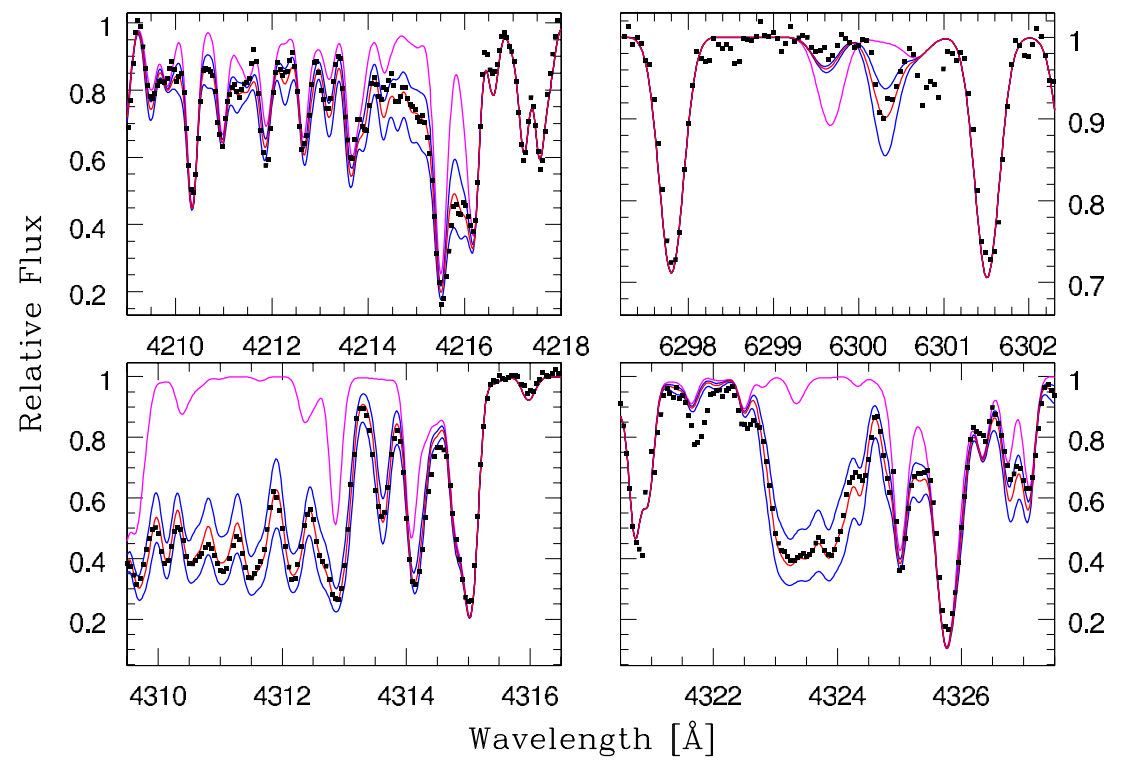

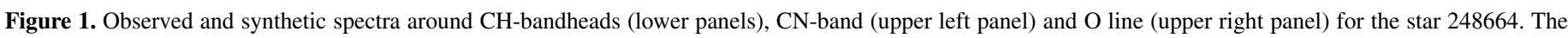

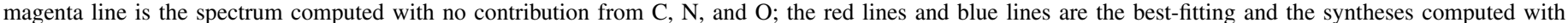
abundance altered by \pm 0.25 dex from the best value, respectively.

(A color version of this figure is available in the online journal.)

responsible for the $\mathrm{He}$ enrichment and for $\mathrm{Na}-\mathrm{O}$ anticorrelations. If, alternatively, the $s$-process elements are produced in the weak component by massive stars that explode as Type II supernovae, it remains to be seen whether the models can produce elements as heavy as $\mathrm{Ba}$ and $\mathrm{La}$ in stellar environments characterized by the chemical abundances observed in $\omega$ Cen.

An important ingredient to understand the star formation history of this poorly understood GC is the determination of the relative ages of its stellar populations. Numerous studies based on CMD analysis combined with metallicity distribution of turnoff (TO) and SGB stars have yielded conflicting results, suggesting age differences from less than 2 Gyr (Lee et al. 2005; Sollima et al. 2005; Calamida et al. 2009), up to $5 \mathrm{Gyr}$ (Villanova et al. 2007). Rapid-formation scenarios wherein the entire cluster could have formed within a few times $10^{8}$ years have recently been suggested by D'Antona et al. (2011) and Valcarce \& Catelan (2011).

Theoretical isochrones show that a variation of the total $\mathrm{C}+\mathrm{N}+\mathrm{O}$ abundance can have an effect on the GC ages obtained from CMD fitting. Therefore, the relative age dating of the stellar populations hosted in the cluster would be severely affected by the presence of $\mathrm{C}+\mathrm{N}+\mathrm{O}$ differences among the $\omega$ Cen subpopulations (Cassisi et al. 2008; Ventura et al. 2009; Pietrinferni et al. 2009; D'Antona et al. 2009).

In an effort to shed light on these issues, in this paper we measure the overall $\mathrm{C}+\mathrm{N}+\mathrm{O}$ abundance in $77 \mathrm{RGB}$ stars of $\omega$ Cen, which span nearly its entire metallicity range. The layout of this paper is as follows. In Section 2 we describe the data analysis; results are presented in Section 3, and their impact on the chemical enrichment scenario and the age measurements are discussed in Sections 4 and 5, respectively; Section 6 is a summary of our results.

\section{OBSERVATIONS AND DATA REDUCTION}

Our data set consists in a sample of 77 RGB stars observed with the FLAMES/GIRAFFE HR4 setup (program: 082.D0424A). We also have, from Marino et al. (2011a), additional spectra for the same stars with different GIRAFFE setups, from which we have derived abundances for $\mathrm{Fe}, \mathrm{Na}, \mathrm{O}$, and $n$-capture element La (75\% s-process in the solar system; Simmerer et al. 2004). In Marino et al. (2011a) we provide measurements also for Ba abundances, but here we prefer to use only $\mathrm{La}$ as representative of $n$-capture elements because $\mathrm{Ba}$ measurements are more uncertain, since the only analyzed Ba transition is a blend (see Marino et al. 2011a). We refer the reader to this paper for a more detailed description of the sample and the data reduction. Here, we analyze, for 77 stars of the Marino et al. (2011a) sample, chemical abundances for carbon and nitrogen.

The HR4 setup covers the spectral range from $\sim 4188$ to $\sim 4392 \AA$, providing a resolution $R \sim 20,000$. The typical signal-to-noise ratio of the final combined spectra at the central wavelength of the spectral range is $\sim 80$.

Our abundance analysis used the local thermodynamic equilibrium analysis code MOOG (Sneden 1973). Carbon was measured from spectral synthesis of the $G$ bandheads $\left(A^{2} \Delta-X^{2} \Pi\right)$ near 4314 and $4323 \AA$. The nitrogen abundance was derived from synthesis of the $\mathrm{CN}$ blue-system $\left(B^{2} \Sigma-X^{2} \Sigma\right)$ bandhead at $\sim 4215 \AA$. The synthesis line list for the blue $\mathrm{CN}$ band is described in Hill et al. (2002). The line list for the $\mathrm{CH}$ band was provided by B. Plez (2008, private communication). Synthetic spectra were employed by using Castelli \& Kurucz (2004) models for effective temperatures $\left(T_{\text {eff }}\right)$, gravities $(\log g)$, metallicities, and microturbulences $\left(\xi_{t}\right)$ determined in Marino et al. (2011a). In computing the $\mathrm{C}$ abundance we used the previously determined O contents (Marino et al. 2011a), and for N, both observed $\mathrm{C}$ and $\mathrm{O}$ abundances needed to be employed. As an example, we show in Figure 1 the spectral synthesis for the $\mathrm{CH}$ and $\mathrm{CN}$ bands, and the $\mathrm{O}$ line for star 248664. For more details on the atmospheric-parameter determinations, and a discussion on related uncertainties, we refer to Marino et al. (2011a). Here, we focus on the $\mathrm{C}$ and $\mathrm{N}$ measurements.

The error analysis for $\mathrm{C}$ and $\mathrm{N}$ was performed by varying $T_{\text {eff }}, \log g$, metallicity, and $\xi_{\mathrm{t}}$ for several stars, and redetermining the abundances. The parameters were varied by $\Delta T_{\text {eff }}=$ $\pm 100 \mathrm{~K}, \Delta \log g= \pm 0.20, \Delta \xi_{\mathrm{t}}= \pm 0.10 \mathrm{~km} \mathrm{~s}^{-1}$, and metallicity 

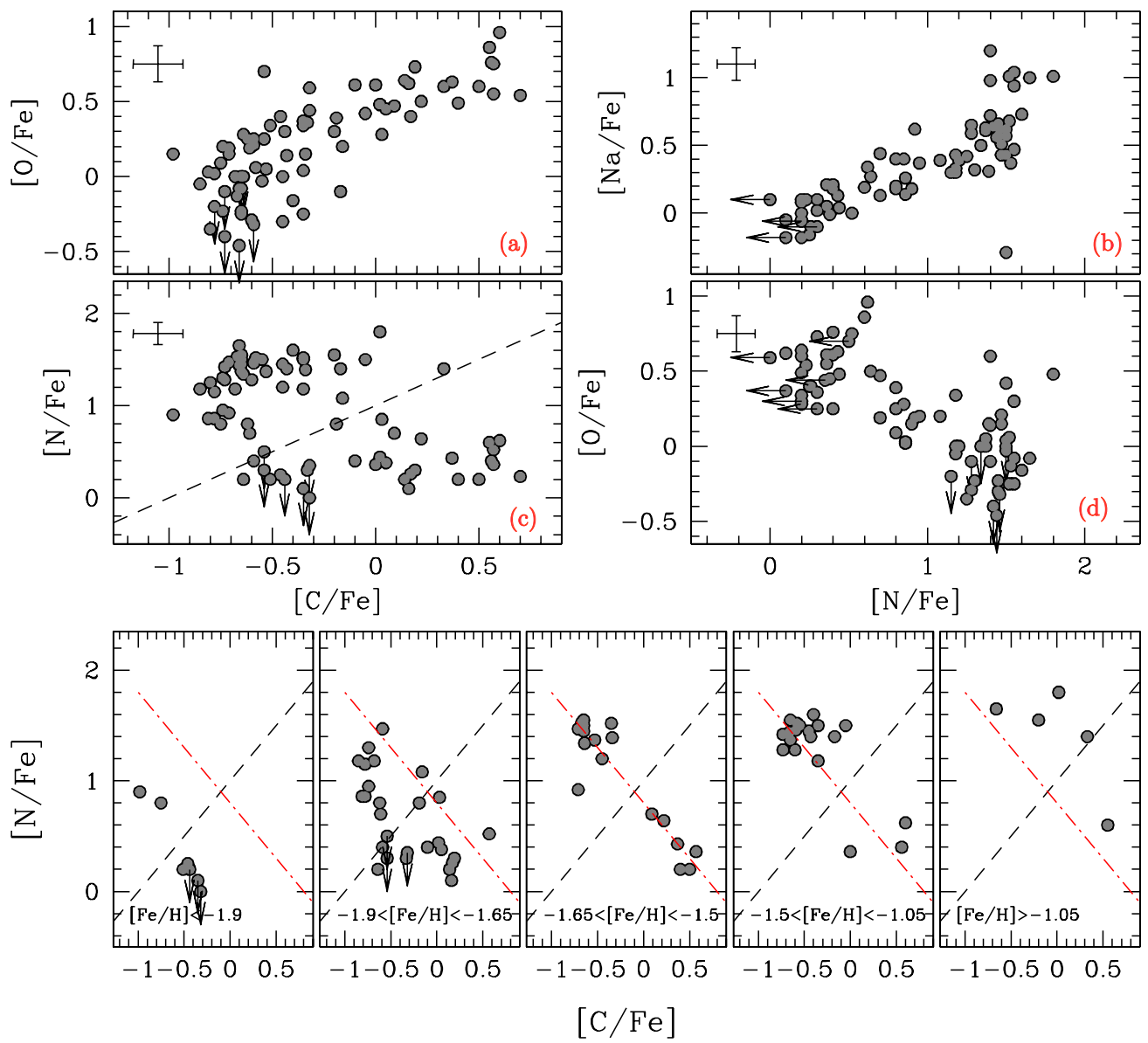

Figure 2. Upper panels: $[\mathrm{N} / \mathrm{Fe}]$ and $[\mathrm{O} / \mathrm{Fe}]$ vs. $[\mathrm{C} / \mathrm{Fe}]$ (panels (c), (a)), and $[\mathrm{O} / \mathrm{Fe}]$ and $[\mathrm{Na} / \mathrm{Fe}]$ vs. $[\mathrm{N} / \mathrm{Fe}]($ panels (d), (b)). The dashed black line in panel (c) separates C-poor/N-rich stars from $\mathrm{C}$-rich/N-poor ones. Lower panels: $[\mathrm{N} / \mathrm{Fe}]$ vs. $[\mathrm{C} / \mathrm{Fe}]$ in metallicity bins defined in Marino et al. (2011a). In each panel, the dashed black line is the same as in panel (c), the red dot-dashed line represents the "best-fit" line of constant $[\mathrm{C} / \mathrm{Fe}]+[\mathrm{N} / \mathrm{Fe}](=0.80)$ for the mid-metallicity stars.

(A color version of this figure is available in the online journal.)

by 0.10 dex. In addition, the derived $\mathrm{C}$ abundance is dependent on the $\mathrm{O}$ abundance and therefore so is the $\mathrm{N}$ abundance, and the circulation of correlated errors tends to mimic the effects of CNO-cycle burning (e.g., Iben \& Renzini 1983). Indeed, in the red giant atmospheres carbon is mainly present in the $\mathrm{CO}$ molecules, and in the molecular equilibrium an overestimation in oxygen produces an overestimation of carbon from the $\mathrm{CH}$ band, and vice versa. In the $\mathrm{CN}$ bands, a carbon overestimation causes nitrogen to be underestimated. In the treatment of the internal errors for carbon, we then varied the oxygen abundance by \pm 0.10 and repeated the spectrum synthesis to determine the error introduced by uncertainties in the $\mathrm{O}$ abundances. These errors were then added in quadrature to the errors introduced by atmospheric parameters and yielded an overall error of $\sim \pm 0.10$ dex to the $[\mathrm{C} / \mathrm{Fe}]$ abundances. For nitrogen we varied both $\mathrm{O}$ and $\mathrm{C}$, the latter by $\pm 0.10 \mathrm{dex}$. We added these errors in quadrature with those introduced by the model atmosphere and estimated the internal uncertainty of the $[\mathrm{N} / \mathrm{Fe}]$ values to be $\sim 0.20-0.30$.

Carbon and nitrogen abundances for $\omega$ Cen RGB stars were determined by Brown \& Wallerstein (1993; 6 stars), Norris \& Da Costa (1995; 40 stars), and Stanford et al. (2010; 33 stars). A proper comparison with these studies cannot be made because there are no stars in common; however, we note that our $\mathrm{C}$ and $\mathrm{N}$ values span a range similar to that of Brown \& Wallerstein (1993) and Stanford et al. (2010). Possible systematic differences in $\mathrm{C}$ could not be excluded between our stars and those of Stanford et al., while systematically lower N abundances (of $\sim 0.50 \mathrm{dex}$ ) were found by Norris \& Da Costa (1995).

In Marino et al. (2011a), we derived the O abundance from the line at $6300 \AA$ by comparing our observations with synthetic spectra that had solar C and N. Here, we redetermine oxygen by adopting in the synthesis the measured $\mathrm{N}$ and $\mathrm{C}$ abundances. These improved $[\mathrm{O} / \mathrm{Fe}]$ measures are in good agreement with the previous measurements, with only few stars differing by more than 0.1 dex from the values in Marino et al. (2011a).

\section{RESULTS}

Figure 2 plots the abundances of $\mathrm{C}, \mathrm{N}$, and $\mathrm{O}$ (Table 1) measured in this paper and the Na measurements from Marino et al. (2011a). We observe large star-to-star variations in all of these elements, as has already observed in all the GCs studied to date. Carbon spans a range from $[\mathrm{C} / \mathrm{Fe}] \sim-0.9$ to $[\mathrm{C} / \mathrm{Fe}] \sim$ +0.6 . Most stars are $\mathrm{C}$-depleted, but there is a population of stars with $[\mathrm{C} / \mathrm{Fe}]>0.0$. Nitrogen spans almost 2 dex, from $[\mathrm{N} / \mathrm{Fe}] \sim 0.0$ to stars with $[\mathrm{N} / \mathrm{Fe}] \sim 1.8$ dex. There is only one star (214842) that is enhanced in both $\mathrm{C}$ and $\mathrm{N}$ (Figure 2, panel c).

Our results show the existence of well-defined patterns among $\mathrm{C}, \mathrm{N}, \mathrm{O}$, and $\mathrm{Na}$. A positive correlation is present between $\mathrm{O}$ and $\mathrm{C}$, and between $\mathrm{Na}$ and $\mathrm{N}$, and $\mathrm{N}$ anticorrelates with $\mathrm{O}$ 
Table 1

Carbon, Nitrogen, and Oxygen Abundances Ratios Relative to Iron for the Analyzed Stars

\begin{tabular}{|c|c|c|c|}
\hline ID & {$[\mathrm{C} / \mathrm{Fe}]$} & {$[\mathrm{N} / \mathrm{Fe}]$} & {$[\mathrm{O} / \mathrm{Fe}]$} \\
\hline 246266 & -0.68 & 1.18 & 0.00 \\
\hline 251010 & -0.62 & 0.80 & 0.25 \\
\hline 249334 & -0.80 & 1.25 & -0.35 \\
\hline 248664 & -0.19 & 0.80 & 0.39 \\
\hline 240777 & 0.02 & 0.44 & 0.48 \\
\hline 239549 & -0.71 & 0.92 & 0.19 \\
\hline 240160 & -0.78 & 0.86 & 0.02 \\
\hline 241561 & -0.74 & 0.95 & 0.20 \\
\hline 236533 & -0.66 & 1.65 & -0.08 \\
\hline 249427 & -0.65 & 1.50 & -0.35 \\
\hline 245973 & -0.46 & 0.25 & 0.40 \\
\hline 246861 & 0.02 & 1.80 & 0.48 \\
\hline 236180 & -0.43 & 1.40 & 0.14 \\
\hline 239113 & 0.19 & 0.30 & 0.73 \\
\hline 250606 & 0.55 & 0.60 & 0.86 \\
\hline 227946 & -0.81 & 0.86 & 0.03 \\
\hline 120847 & 0.09 & 0.70 & 0.47 \\
\hline 237123 & -0.20 & 1.55 & 0.30 \\
\hline 224515 & -0.54 & 0.50 & 0.70 \\
\hline 225055 & -0.64 & 0.20 & 0.28 \\
\hline 212584 & -0.05 & 1.50 & 0.42 \\
\hline 234159 & 0.60 & 0.62 & 0.96 \\
\hline 214842 & 0.33 & 1.40 & 0.60 \\
\hline 218445 & 0.22 & 0.64 & 0.50 \\
\hline 230340 & -0.66 & 1.44 & -0.46 \\
\hline 224500 & 0.40 & 0.20 & 0.49 \\
\hline 211516 & -0.51 & 0.20 & 0.34 \\
\hline 225246 & -0.61 & 0.70 & 0.19 \\
\hline 229207 & -0.35 & 1.18 & 0.34 \\
\hline 239529 & 0.14 & 0.20 & 0.64 \\
\hline 245199 & -0.78 & 1.15 & -0.20 \\
\hline 245724 & -0.44 & 0.20 & 0.30 \\
\hline 246239 & -0.59 & 1.46 & -0.32 \\
\hline 242745 & -0.35 & 0.10 & 0.37 \\
\hline 231867 & 0.56 & 0.40 & 0.76 \\
\hline 236988 & -0.60 & 1.28 & -0.29 \\
\hline 117319 & -0.33 & 0.30 & 0.36 \\
\hline 218472 & -0.32 & 0.00 & 0.59 \\
\hline 217404 & -0.35 & 1.52 & -0.25 \\
\hline 216985 & 0.16 & 0.10 & 0.62 \\
\hline 213225 & -0.45 & 1.45 & -0.30 \\
\hline 220325 & -0.45 & 1.20 & 0.00 \\
\hline 232599 & -0.59 & 0.40 & 0.25 \\
\hline 230502 & -0.75 & 0.80 & 0.09 \\
\hline 222357 & -0.35 & 1.50 & 0.04 \\
\hline 249670 & -0.32 & 0.35 & 0.44 \\
\hline 246585 & -0.65 & 1.55 & -0.25 \\
\hline 236112 & -0.54 & 0.30 & 0.25 \\
\hline 244812 & -0.17 & 1.40 & -0.10 \\
\hline 240679 & 0.05 & 0.38 & 0.45 \\
\hline 217650 & -0.59 & 1.47 & 0.21 \\
\hline 215367 & -0.98 & 0.90 & 0.15 \\
\hline 227198 & -0.65 & 1.37 & 0.00 \\
\hline 222732 & -0.58 & 1.52 & 0.06 \\
\hline 222298 & -0.67 & 1.53 & -0.13 \\
\hline 236813 & -0.65 & 1.45 & -0.23 \\
\hline 328201 & -0.55 & 1.50 & -0.03 \\
\hline 245870 & -0.10 & 0.40 & 0.61 \\
\hline 246416 & -0.64 & 1.34 & 0.00 \\
\hline 239444 & -0.73 & 1.42 & -0.40 \\
\hline 241359 & 0.17 & 0.26 & 0.40 \\
\hline 241046 & 0.37 & 0.43 & 0.63 \\
\hline 241447 & 0.70 & 0.23 & 0.54 \\
\hline 236354 & -0.34 & 1.39 & 0.15 \\
\hline 232373 & 0.57 & 0.36 & 0.55 \\
\hline
\end{tabular}

Table 1

(Continued)

\begin{tabular}{lrcr}
\hline \hline ID & {$[\mathrm{C} / \mathrm{Fe}]$} & {$[\mathrm{N} / \mathrm{Fe}]$} & {$[\mathrm{O} / \mathrm{Fe}]$} \\
\hline 230070 & -0.40 & 1.60 & -0.16 \\
230804 & -0.65 & 1.55 & -0.08 \\
226863 & -0.16 & 1.08 & 0.20 \\
225658 & 0.57 & 0.52 & 0.75 \\
228375 & 0.00 & 0.36 & 0.61 \\
215912 & 0.50 & 0.20 & 0.60 \\
224533 & -0.85 & 1.18 & -0.05 \\
237327 & -0.73 & 1.28 & -0.10 \\
232639 & -0.74 & 1.30 & -0.23 \\
240754 & -0.53 & 1.37 & 0.05 \\
217039 & -0.71 & 1.47 & 0.15 \\
226236 & 0.03 & 0.85 & 0.28 \\
\hline
\end{tabular}

(Figure 2, panels (a), (b), (d)). A C-N anticorrelation is not obvious (panel c), but it can be more easily recognizable by grouping stars on the basis of different Fe content, as will be discussed in Section 3.1. Such chemical patterns indicate the occurrence of high-temperature H-burning involving the $\mathrm{CNO}$ and $\mathrm{NeNa}$ cycles, and are consistent with prediction in Ventura \& D'Antona (2009).

In the following sections, we present the pattern of $\mathrm{C}, \mathrm{N}$, $\mathrm{O}$ abundances with $[\mathrm{Fe} / \mathrm{H}]$ (Section 3.1) and with respect to the position of stars on the $\mathrm{Na}-\mathrm{O}$ anticorrelation (Section 3.2) studied by Marino et al. (2011a).

\section{1. $\mathrm{CNO}$ Pattern with $[\mathrm{Fe} / \mathrm{H}]$}

Before discussing in detail the $\mathrm{C}, \mathrm{N}, \mathrm{O}$ patterns, we recall that $\omega$ Cen exhibits a large spread in $[\mathrm{Fe} / \mathrm{H}]$, ranging from $\sim-2.0$ to $\sim-0.7$ dex. The iron distribution is multi-modal with distinct peaks at $[\mathrm{Fe} / \mathrm{H}] \sim-1.75,-1.60,-1.45,-1.00$, and there is a broad distribution of stars extending up to $[\mathrm{Fe} / \mathrm{H}] \sim-0.70$ (Marino et al. 2011a). Although the existence of these multiple populations is clear, observational errors often prevent us from definitively establishing which individual stars belong to which population. Keeping this in mind, Marino et al. (2011a) defined six groups of stars with different iron abundances to investigate the $\mathrm{Na}-\mathrm{O}$ anticorrelation for different metallicity groups (see their Figure 8).

In the lower panels of Figure 2, we perform a similar analysis for $\mathrm{C}$ versus $\mathrm{N}$ in the same metallicity bins defined in Marino et al. (2011a). Unfortunately, we do not have C and $\mathrm{N}$ measurements for the most metal-rich group, with $[\mathrm{Fe} / \mathrm{H}]>-0.90$, of Marino et al. (2011a). We see that the $\mathrm{C}-\mathrm{N}$ anticorrelation is present across the entire range of metallicities. The dashed-black line in each panel (that is the same as in panel c) has been traced to arbitrarily separate the C-poor/N-rich population from the $\mathrm{C}$-rich/N-poor one. The red dot-dashed line is the "best-fit" line of constant $[\mathrm{C} / \mathrm{Fe}]+[\mathrm{N} / \mathrm{Fe}]$ for the midmetallicity stars, that has been taken equal to 0.80 . It is worth noting that when we compare the relative location along the $\mathrm{C}-\mathrm{N}$ anticorrelation with this line, it becomes evident that the average $\mathrm{C}$ abundance increases from lower to higher metallicities. The shape of the $\mathrm{C}-\mathrm{N}$ anticorrelation for different metallicities is similar to that of the $\mathrm{Na}-\mathrm{O}$ anticorrelation (Figure 8 of Marino et al. 2011a), with the most metal-rich groups hosting a larger fraction of N-rich (Na-rich, O-C-poor) stars. This behavior leads few stars, belonging to the metal richer group (e.g., 214842), to be enhanced both in $\mathrm{C}$ and $\mathrm{N}$.

In the upper panels of Figure 3, we plot the individual carbon, nitrogen, and oxygen abundances against iron. 

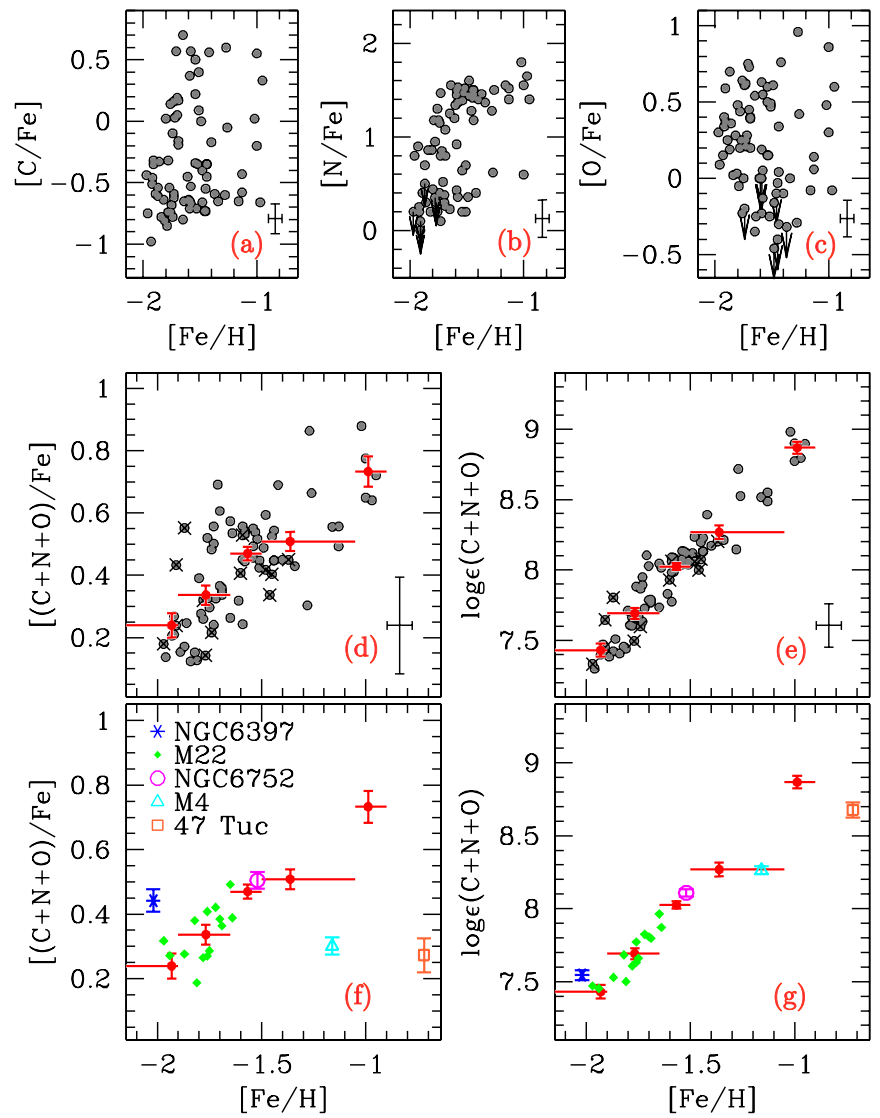

Figure 3. Upper panels: $[\mathrm{C} / \mathrm{Fe}],[\mathrm{N} / \mathrm{Fe}]$, and $[\mathrm{O} / \mathrm{Fe}]$ vs. $[\mathrm{Fe} / \mathrm{H}]$. Middle panels: $[(\mathrm{C}+\mathrm{N}+\mathrm{O}) / \mathrm{Fe}]$ and $\log \epsilon(\mathrm{C}+\mathrm{N}+\mathrm{O})$ vs. $[\mathrm{Fe} / \mathrm{H}]$. Stars with upper limits for $\mathrm{O}$ or $\mathrm{N}$ abundances are represented as gray crosses. Red points represent the average $\mathrm{C}+\mathrm{N}+\mathrm{O}$ content in the metallicity intervals spanned by the horizontal red bars. Vertical bars are the errors associated with the mean values. Lower panels: average $[(\mathrm{C}+\mathrm{N}+\mathrm{O}) / \mathrm{Fe}]$ vs. iron for the GCs quoted in the inset compared with the $\omega$ Cen CNO mean values (red points) of panels (d) and (e). Data for the mono-metallicity GCs are from Ivans et al. (1999) for M4, and Carretta et al. (2005) for 47 Tuc, NGC 6752, and NGC 6397. Data for M22 are from Marino et al. (2011b).

(A color version of this figure is available in the online journal.)

While $[\mathrm{N} / \mathrm{Fe}]$ exhibits a mild correlation with $[\mathrm{Fe} / \mathrm{H}]$, there is no clear correlation either between $[\mathrm{C} / \mathrm{Fe}]$ and $[\mathrm{Fe} / \mathrm{H}]$ or between $[\mathrm{O} / \mathrm{Fe}]$ and $[\mathrm{Fe} / \mathrm{H}]$. A significant spread in light elements is present at all metallicities with $[\mathrm{C} / \mathrm{Fe}],[\mathrm{N} / \mathrm{Fe}]$, and $[\mathrm{O} / \mathrm{Fe}]$ spanning large ranges (more than $1 \mathrm{dex}$ ).

If, however, we instead plot the total $[\mathrm{C}+\mathrm{N}+\mathrm{O}]$ against $[\mathrm{Fe} / \mathrm{H}]$, we find a correlation with a Pearson coefficient of 0.71 . This is shown in panel (d) for the abundance relative to iron and in panel (e) for the total abundance. The red points show the averages over bins in $[\mathrm{Fe} / \mathrm{H}]$ defined above. The vertical error bars correspond to the error in the mean related to the observed dispersion and the horizontal bars denote the boundaries of the $[\mathrm{Fe} / \mathrm{H}]$ bins.

The representative error bar shown in the lower right of panels (d) and (e) comes from adding the individual $\mathrm{C}, \mathrm{N}$, and $\mathrm{O}$ errors in quadrature, and indicates that the error in the total $[(\mathrm{C}+\mathrm{N}+\mathrm{O}) / \mathrm{Fe}]$ should be quite large $(\sim 0.3 \mathrm{dex})$. When we estimated the errors in these abundances, we made the assumption that the abundances were statistically independent. Given, though, that the $\mathrm{C}, \mathrm{N}$, and $\mathrm{O}$ abundances are correlated with each other, a more realistic estimate of the error might be derived empirically from the rms of the $[(\mathrm{C}+\mathrm{N}+\mathrm{O}) / \mathrm{Fe}]$ within each metallicity bin. In this case, we find an rms ranging from 0.10 to 0.15 dex, and stars with intermediate metallicity $(-1.65 \leqslant$ $[\mathrm{Fe} / \mathrm{H}]<-1.05)$ have the largest rms.

The bottom panels of Figure 3 show the trends we have derived for $\omega$ Cen in the context of other clusters. M22 has stars with a range of metallicities (Marino et al. 2009), and these stars are shown as the green points, which happen to follow the $\omega$ Cen trend almost perfectly. On the other hand, the mono-metallic clusters for which we have data (NGC 6397, NGC 6752, M4, and 47 Tuc) do not follow this trend and, in general, show no discernible trend of $[(\mathrm{C}+\mathrm{N}+\mathrm{O}) / \mathrm{Fe}]$ with metallicity.

\section{2. $\mathrm{CNO}, \mathrm{Na}$, and La Abundances in the $\mathrm{Na}-\mathrm{O}$ Anticorrelation}

To investigate how the studied chemical abundances behave for stars occupying a different location on the $\mathrm{Na}-\mathrm{O}$ plane, we divided stars into two groups based on their location along the $\mathrm{Na}-\mathrm{O}$ trend (Marino et al. 2011a). In normal mono-metallic clusters different generations of stars can be segregated in this way, and the $\mathrm{Na}-\mathrm{O}$ groupings tend to have different $\mathrm{C}$ and $\mathrm{N}$ abundances, as can be seen in the case of M4 (Marino et al. 2008, see their Figure 10). The situation in $\omega$ Cen has an added level of complexity, since the large variation in metallicity does not allow us to simply identify two (or more) populations via $\mathrm{Na}$ and $\mathrm{O}$. Both Johnson \& Pilachowski (2010) and Marino et al. (2011a) have shown that stars of both first and second generations are present across a large range of metallicities. Note that here we extend the same nomenclature used for mono-metallic GCs to $\omega$ Cen and name first and second generation Na-poor/O-rich and Na-rich/O-poor stars, respectively, whatever their $[\mathrm{Fe} / \mathrm{H}]$. Of course, in the case of $\omega$ Cen this designation should not be taken literally, as stars of both groups are present in a large range in metallicity. We will therefore first follow the chemical patterns for the elements affected by $p$-captures $(\mathrm{C}, \mathrm{N}, \mathrm{O}, \mathrm{Na})$, $n$-capture elements $(\mathrm{La})$, and $\mathrm{C}+\mathrm{N}+\mathrm{O}$ for the first generation alone, and then will examine the second generation.

In Figure 4, we represent in different colors our selected first- and second-generation stars on the $\mathrm{Na}-\mathrm{O}$ anticorrelation from Marino et al. (2011a). The Na-poor/O-rich firstgeneration (green open triangles) and the $\mathrm{Na}$-rich/O-poor second-generation stars (magenta open squares) have been arbitrarily selected by the dashed line. Similarly to that done in the lower panels of Figure 2, stars have been represented in different panels depending on their $[\mathrm{Fe} / \mathrm{H}]$ bin. In each panel, the gray crosses represent the entire sample. The so selected first- and second-generation stars have been plotted in an O-La plane, as shown in Figure 5 (left panel). It is worth noting that in first-generation stars $[\mathrm{O} / \mathrm{Fe}]$ abundances correlate with $[\mathrm{La} / \mathrm{Fe}]$, with the Pearson coefficient equal to 0.73 . To quantify this correlation we have determined the mean $[\mathrm{O} / \mathrm{Fe}]$ abundances for three La intervals spanned by first-generation stars, whose sizes are indicated by the horizontal dark-green lines in Figure 5. The mean $[\mathrm{O} / \mathrm{Fe}]$ values for the different $\mathrm{Fe}$ and La intervals are listed in Table 2, together with the rms and associated errors. In first stellar generation, some hints for a correlation may be present also between $[\mathrm{O} / \mathrm{Fe}]$ and $[\mathrm{Fe} / \mathrm{H}]$, as shown in the middle panel of Figure 5. In this case, the $\mathrm{O}$ rise is more difficult to be claimed over observational errors and the mean $[\mathrm{O} / \mathrm{Fe}]$ values in the selected metallicity bins (shown in dark-green in the middle panel of Figure 5) agree within a $3 \sigma$ values.

We suggest some caution with the $\mathrm{O}$ trend in first-generation stars, as we cannot fully exclude that it may be due to some unidentified systematics with iron. Johnson \& Pilachowski (2010) do not find a trend of $[\mathrm{O} / \mathrm{Fe}]$ with $[\mathrm{Fe} / \mathrm{H}]$, but claim 


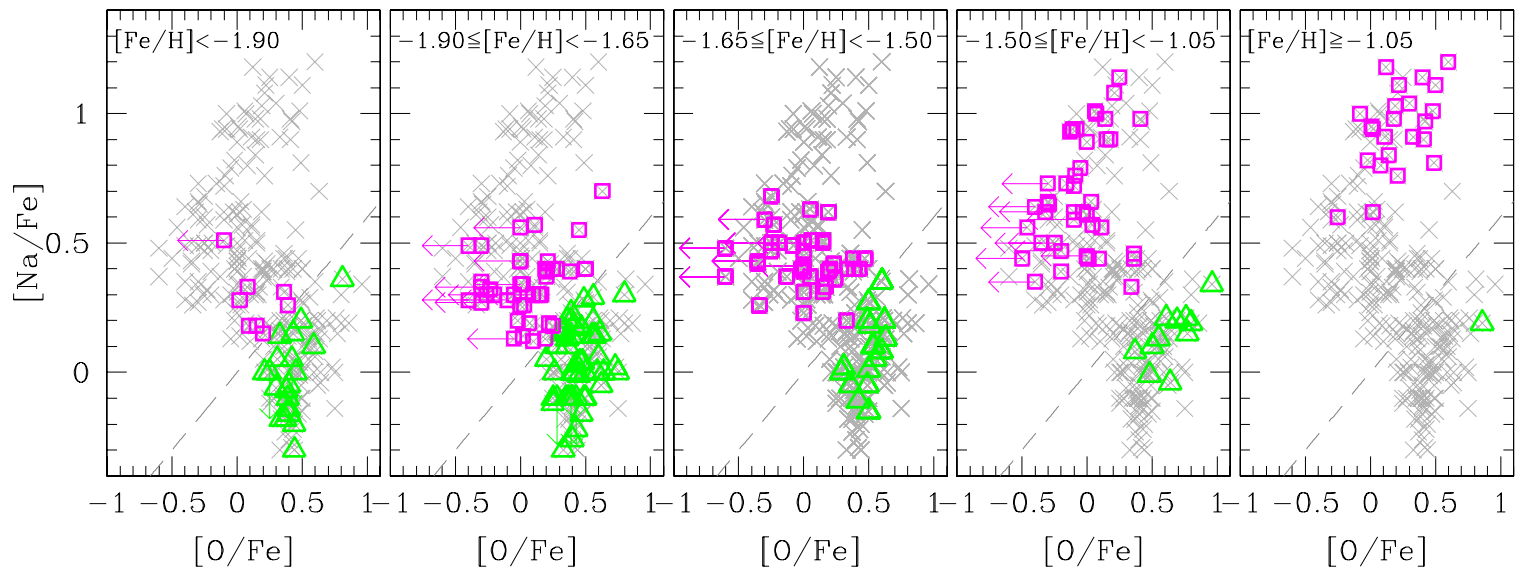

Figure 4. Our adopted division of stars in O-rich/Na-poor (first generation) and O-poor/Na-rich ones (second generation) has been represented in the Na-O plane for each metallicity bin, as quoted in the insets. The dashed line separates the selected first-generation stars represented by green triangles, and from the selected second-generation stars represented by magenta squares. Gray crosses in each panel represent the entire sample analyzed by Marino et al. (2011a).

(A color version of this figure is available in the online journal.)

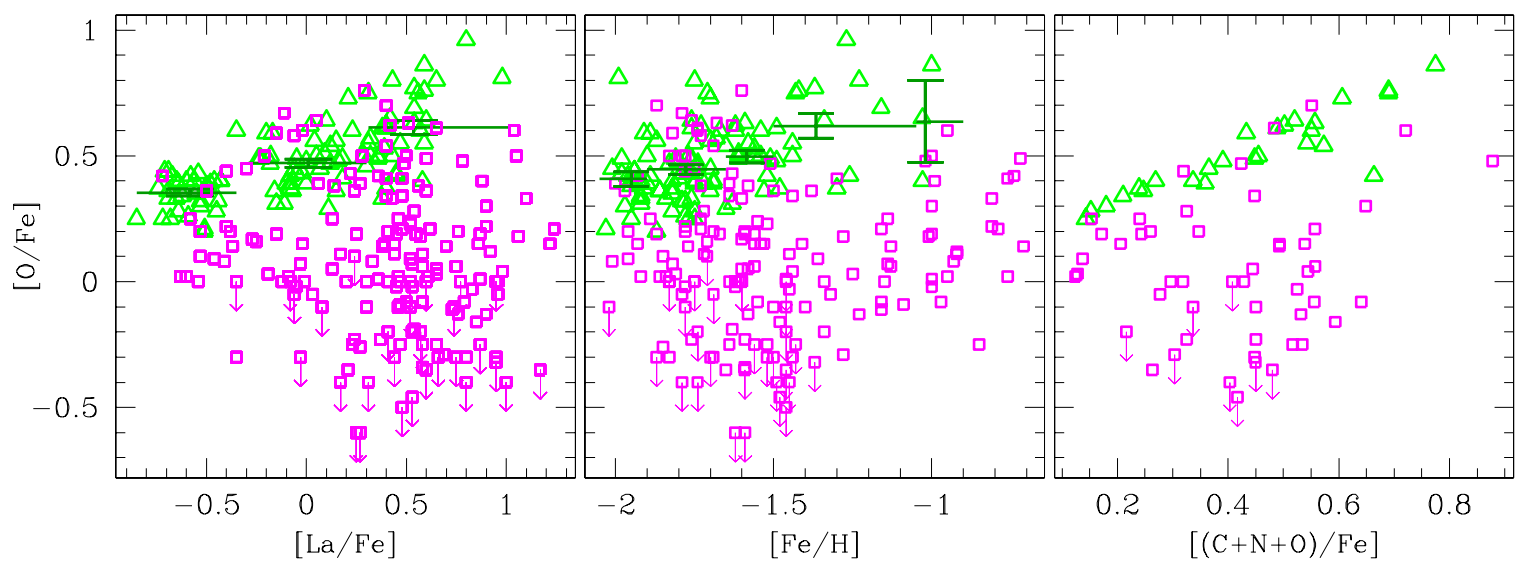

Figure 5. $\mathrm{O}$ abundance ratios as a function of $[\mathrm{La} / \mathrm{Fe}],[\mathrm{Fe} / \mathrm{H}]$, and $[(\mathrm{C}+\mathrm{N}+\mathrm{O}) / \mathrm{Fe}]$. Symbols are the same as in Figure 4 . The dark green vertical error bars represent the error associated with the mean $[\mathrm{O} / \mathrm{Fe}]$ abundance in different intervals in $[\mathrm{La} / \mathrm{Fe}]$ and $[\mathrm{Fe} / \mathrm{H}]$ delimited by the horizontal line.

(A color version of this figure is available in the online journal.)

Table 2

Oxygen Mean Content for First Generation Stars in the Fe Bins of Marino et al. (2011a) and for Three Intervals in [La/Fe]

\begin{tabular}{|c|c|c|c|c|c|c|c|c|c|}
\hline & \multicolumn{6}{|c|}{$[\mathrm{Fe} / \mathrm{H}]$ Range } & \multicolumn{3}{|c|}{$[\mathrm{La} / \mathrm{Fe}]$ Range } \\
\hline & $<-1.90$ & $-1.90 /-1.65$ & $-1.65 /-1.50$ & $-1.50 /-1.05$ & $-1.05 /-0.95$ & $>-0.95$ & $<-0.30$ & $-0.30 / 0.30$ & $>0.30$ \\
\hline$[\mathrm{O} / \mathrm{Fe}]$ & 0.41 & 0.45 & 0.50 & 0.62 & 0.64 & $\cdots$ & 0.35 & 0.47 & 0.61 \\
\hline \pm & 0.03 & 0.02 & 0.03 & 0.05 & 0.16 & $\cdots$ & 0.01 & 0.02 & 0.03 \\
\hline$\sigma$ & 0.13 & 0.13 & 0.10 & 0.18 & 0.23 & $\ldots$ & 0.08 & 0.10 & 0.15 \\
\hline First-generation stars & 20 & 48 & 17 & 14 & 3 & 0 & 33 & 38 & 31 \\
\hline
\end{tabular}

instead to find weak trends $(\sim 0.1 \mathrm{dex})$ in other $\alpha$ element ratios, such as $[\mathrm{Ca} / \mathrm{Fe}]$ and $[\mathrm{Si} / \mathrm{Fe}]$, which however are even weaker than our trend in $[\mathrm{O} / \mathrm{Fe}]$. Note that such trend is detectable only among first-generation stars, as oxygen has been depleted in second-generation stars by $p$-capture reactions.

As shown in the right panel of Figure 5, in first-generation stars $\mathrm{O}$ increases in concert with the total CNO. At odds with first-generation stars, the second generation ones (represented in magenta) do not appear to show any correlation with either La, $\mathrm{Fe}$, and $\mathrm{CNO}$, and occupy a spread region in all these abundance planes. Lanthanum abundance ratios follow a similar pattern for first- and second-generation stars as shown in Figure 6. This implies that the material out of which second-generation stars have formed was not exposed to neutron sources (no additional $n$-captures besides those experienced by first-generation stars) but only to $p$-captures. If AGB stars were responsible for the $p$-capture processing, this must have taken place in stars experiencing negligible third dredge-up, otherwise the material would have been further enriched in $s$-process elements.

In Figure 7, we summarize our results for $\mathrm{C}, \mathrm{N}, \mathrm{O}$, and $\mathrm{Na}$ abundance ratios, which have been plotted as a function of $[(\mathrm{C}+\mathrm{N}+\mathrm{O}) / \mathrm{Fe}],[\mathrm{Fe} / \mathrm{H}]$, and $[\mathrm{La} / \mathrm{Fe}]$, for the first- and secondgeneration stars. Carbon; as well as $\mathrm{O}$, increases as a function of the total $\mathrm{CNO}$ and $[\mathrm{La} / \mathrm{Fe}]$, suggesting that $\mathrm{C}$ and $\mathrm{O}$ evolve in a similar way (though their errors are correlated).

At odds with $\mathrm{C}$ and $\mathrm{O}$, neither $\mathrm{N}$ nor $\mathrm{Na}$ show any evidence for correlation with the total $\mathrm{CNO}$ abundance in first-generation stars. However, the minimum values for these two elements may 


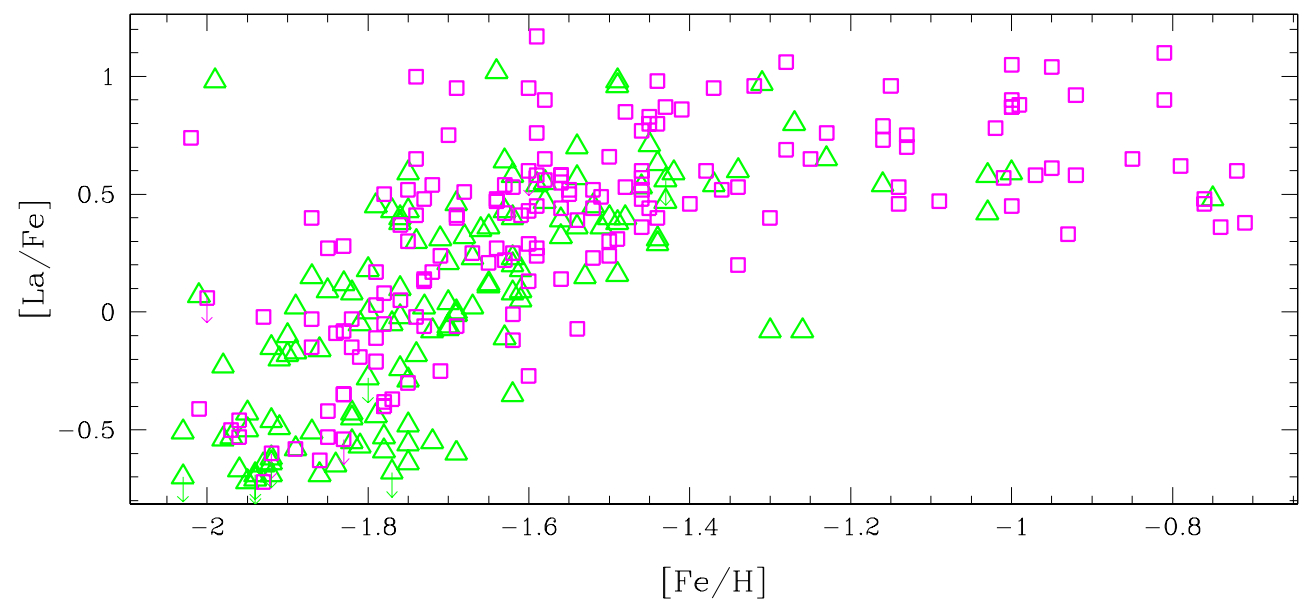

Figure 6. $[\mathrm{La} / \mathrm{Fe}]$ as a function of $[\mathrm{Fe} / \mathrm{H}]$. Symbols are the same as in Figure 4.

(A color version of this figure is available in the online journal.)
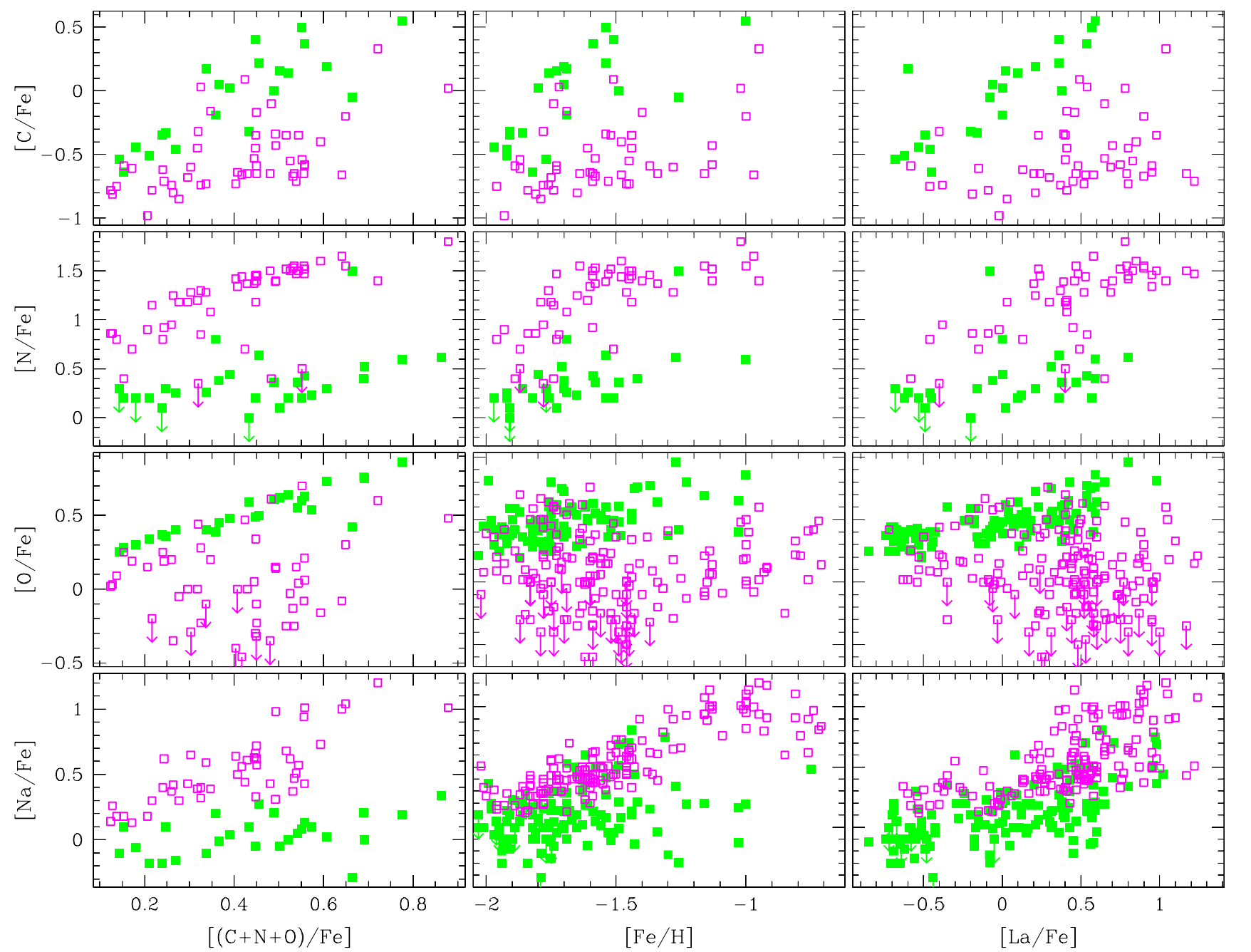

Figure 7. $\mathrm{C}, \mathrm{N}, \mathrm{O}$, and $\mathrm{Na}$ abundance ratios as a function of $[(\mathrm{C}+\mathrm{N}+\mathrm{O}) / \mathrm{Fe}],[\mathrm{Fe} / \mathrm{H}]$, and $[\mathrm{La} / \mathrm{Fe}]$. Symbols are the same as in Figure 4.

(A color version of this figure is available in the online journal.)

increase slightly with both $\mathrm{Fe}$ and $\mathrm{La}$. $\mathrm{N}$ and $\mathrm{Na}$ display a much stronger trend with either the overall $\mathrm{CNO}$, iron, and lanthanum for second-generation stars, i.e., $\mathrm{N}$ and $\mathrm{Na}$ are higher for stars with higher $\mathrm{CNO} / \mathrm{Fe} / \mathrm{La}$.

All the observed abundance pattern suggests that $\mathrm{C}$ and $\mathrm{O}$ from one side, and $\mathrm{N}$ and $\mathrm{Na}$ form the other, have undergone a similar processing in the evolution of the cluster. In addition, the first and second generation, selected on the basis of their $\mathrm{Na}$ and $\mathrm{O}$ content, appear to show well-defined individual chemical trends along the overall observed range in metallicity.

Finally, the CNO increase among first-generation stars is driven by the rise of $\mathrm{O}$ (and partly also of $\mathrm{C}$ ), rather than 

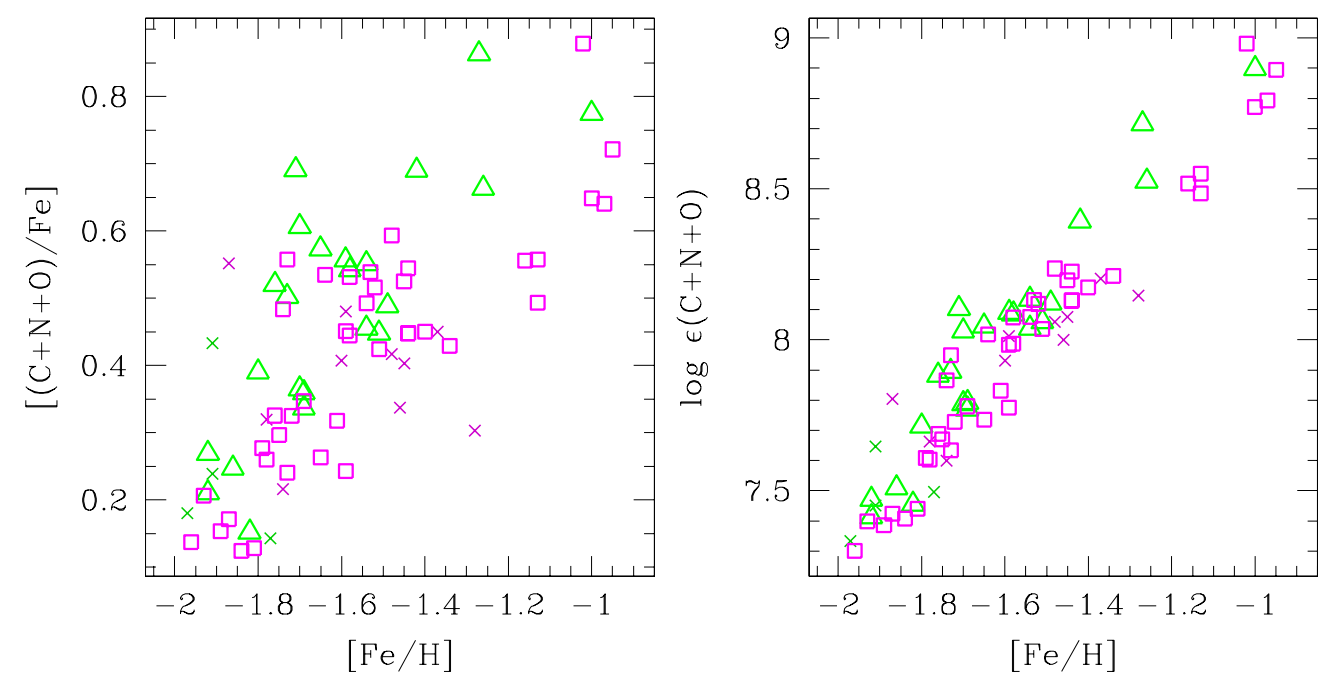

Figure 8. $\mathrm{CNO}$ relative to $\mathrm{Fe}$ and $\log \epsilon(\mathrm{CNO})$ as a function of $[\mathrm{Fe} / \mathrm{H}]$. Symbols are the same as in Figure 4. Crosses mark stars for which we have only an upper limit for $\mathrm{N}$ or $\mathrm{O}$.

(A color version of this figure is available in the online journal.)

$\mathrm{N}$. As $\mathrm{C}$ and $\mathrm{O}$ increase with metallicity, more $\mathrm{N}$ could be produced by $\mathrm{CNO}$ cycling, and so in O-poor/Na-rich second-generation stars both $[\mathrm{N} / \mathrm{Fe}]$ and $[\mathrm{Na} / \mathrm{Fe}]$ increase with metallicity. Figure 8 shows $\mathrm{CNO}$ versus Fe separately for firstand second-generation stars, demonstrating that the increase of $\mathrm{CNO}$ with $\mathrm{Fe}$ is common to both generations. The small offset $(<\sim 0.1$ dex) between the two generations may be real (due to part of CNO elements having been processed to heavier nuclei by $p$-captures), but we cannot fully exclude a slight systematic underestimate of $\mathrm{N}$ among second-generation stars.

\section{THE FORMATION AND CHEMICAL ENRICHMENT OF $\omega$ CEN}

The interpretation of the photometric and spectroscopic evidences on the stellar populations of $\omega$ Cen in terms of its formation and evolution is posing formidable difficulties. In this section, we try to extract as much as possible from what the data themselves apparently demand.

Although there is photometric evidence for at least six different stellar populations in $\omega$ Cen (e.g., Bellini et al. 2010), we have distinguished between a first and a second generation as comprised of those stars that either do not or do show evidence for additional $p$-capture processing at high temperature, respectively. Thus, the first generation may represent a less complex case to interpret in terms of chemical evolution, with the second generation possibly arising from the AGB ejecta of the former one, as generally entertained.

\subsection{Formation Scenarios}

We first explore a scenario in which the progenitor system to $\omega$ Cen has evolved as a single entity, in which the ISM was chemically homogeneous at each time. Thus, one can envisage the first generation to be the result of five or six successive bursts of star formation, with stars in each successive episode being progressively enriched by nucleosynthesis products from corecollapse supernovae from the previous bursts. By construction, the first generation is made up of stars which formed out of an ISM that was not yet polluted by $p$-capture products by AGB stars. This requirement sets an upper limit of 30-40 Myr between the first and the last burst, as later AGB stars would come at play.

Now, before the second generation starts to form, most residual gas must be ejected from the system, thus allowing second-generation stars to be formed out of almost pure AGB ejecta. As emerging from all previous figures, second-generation stars span the full metallicity range of $\omega$ Cen, which suggests that each episode of first-generation formation has produced its own specific contribution to the second generation. Thus, after the five or six initial bursts of star formation, and the ejection of the residual gas, a similar series of bursts should have taken place out of gas from AGB ejecta being accumulated at the bottom of the potential well, via a series of cooling flows.

Also, for this second-generation series of bursts, one can set a time limit to its duration, which comes from the necessity of avoiding the contamination by $\mathrm{C} / \mathrm{O}>1$ materials from carbon star ejecta, which should start some $2 \times 10^{8}$ yr after the very first burst. Therefore, in this scenario, all star formation episodes were confined to within $\sim 2 \times 10^{8}$ yr. Of course, such a scenario with its complex series of events may require still additional complications to explain the presence of the $\mathrm{Na}-\mathrm{O}$ anticorrelation among stars within different individual star formation episodes of the second generation (i.e., within each $[\mathrm{Fe} / \mathrm{H}]$ group). Even more complications may be required if the $\mathrm{Na}-\mathrm{O}$ anticorrelation is due to partial mixing of AGB ejecta with pristine material as often invoked (e.g., D'Ercole et al. 2011 and references therein), though the origin of the diluting material remains to be understood. Five or six successive dilutions with pristine material would ask for an extremely contrived scenario. Alternatively, common-envelope ejecta from intermediate-mass binary stars may offer a less implausible option (Vanbeveren et al. 2011), but it remains to be seen if binaries can provide enough diluting material.

Moreover, as well known, the original first stellar generation must have been much more massive than at present, if enough second-generation stars formed from its AGB ejecta (e.g., Bekki \& Norris 2006; Renzini 2008). This led to the hypothesis of a dwarf galaxy precursor of which $\omega$ Cen would be the stripped, remnant nucleus (e.g., Bekki \& Freeman 2003; D'Ercole et al. 2010). In summary, this scenario formation proceeds through a rapid series of star formation episodes (bursts), separated by just 
few Myr in time, each followed by their own cooling flow made of AGB ejecta to form the second-generation stars in a series of secondary bursts. Tidal stripping will then remove most of the original stellar mass of the system, leaving $\omega$ Cen as the bare nucleus of the original dwarf galaxy.

Alternatively, the several star formation episodes, rather than being sequential in time, may have been separated in space, with each lump of matter having evolved (quasi)independently from the others, before merging together to make the cluster we observe today. In the original dwarf galaxy several high-density sites of star formation were active, each forming a proto-GC of first-generation stars, with each of them later feeding the formation of its own second-generation stars via a cooling flow of AGB-ejecta material. In turn, the densest part of the individual proto-clusters would merge together by dynamical friction and coalesce, thus forming a massive nucleus, to become $\omega$ Cen after all the rest of the dwarf galaxy is tidally stripped.

It is difficult to say whether the former, monolithic scenario is more (or less) contrived than this merger one in which five or six proto-clusters evolve separately before coalescing together. Yet, the stellar population content of this most massive GC in the Galaxy is so complex that we suspect no simple, straightforward model can be found for its formation. Eventually we must admit that we are still far from understanding this most puzzling cluster.

\subsection{Chemical Enrichment}

The puzzle becomes even more difficult when considering the specific chemical patterns exhibited by the stars in the cluster. Here we limit the discussion to the CNO elements.

In Figure 8, $[(\mathrm{C}+\mathrm{N}+\mathrm{O}) / \mathrm{Fe}]$ appears to increase with $[\mathrm{Fe} / \mathrm{H}]$ among first-generation stars, a trend that is dominated by the increase of $[\mathrm{O} / \mathrm{Fe}]$ since oxygen is the most abundant of the three elements. This is also indicated by the tight correlation between $[(\mathrm{C}+\mathrm{N}+\mathrm{O}) / \mathrm{Fe}]$ and $[\mathrm{O} / \mathrm{Fe}]$ seen in the right panel of Figure 5. The possible $[\mathrm{O} / \mathrm{Fe}]$ increase with $[\mathrm{Fe} / \mathrm{H}]$ among first-generation stars is contrary to the decreasing trend shown in all other environments. In fact, $[\mathrm{O} / \mathrm{Fe}]$ decreases in lockstep with $[\mathrm{Fe} / \mathrm{H}]$ in the Galactic thin disk, thick disk, bulge, and halo (e.g., Bensby et al. 2004; Zoccali et al. 2006; Wheeler et al. 1989), a trend that is generally interpreted as due to a prompt enrichment of $\alpha$-element by core collapse supernovae, followed by a slower iron enrichment by Type Ia supernovae (e.g., Matteucci \& Greggio 1986). Moreover, values of [O/Fe] in excess of 0.5 are common among first-generation stars, whereas they are extremely rare in any other environment. Therefore, the normal population in $\omega$ Cen, the one not affected by proton captures, also shows a unique chemical evolution history, not paralleled in any other known environment.

Thus, this classical scheme of chemical evolution may not apply to $\omega$ Cen's first generation, as we have seen that oxygen keeps increasing faster than iron, a clear evidence contrary to Type Ia supernovae playing a role in its enrichment. If so, one has to resort only on core collapse supernovae in trying to explain the $[(\mathrm{C}+\mathrm{N}+\mathrm{O}) / \mathrm{Fe}]$ trend with $[\mathrm{Fe} / \mathrm{H}]$. This is not an easy task.

The existence of a broad range of metallicities among the firstgeneration stars argues for enrichment and star formation having proceeded together in the progenitor object of $\omega$ Cen, though quite possibly as a series of successive bursts, as discussed in the previous subsection and indicated by the distinct photometric sequences present in this cluster (e.g., Bellini et al. 2010) paralleled by a multimodal distribution of $[\mathrm{Fe} / \mathrm{H}]$ (e.g., Marino et al. 2011a).

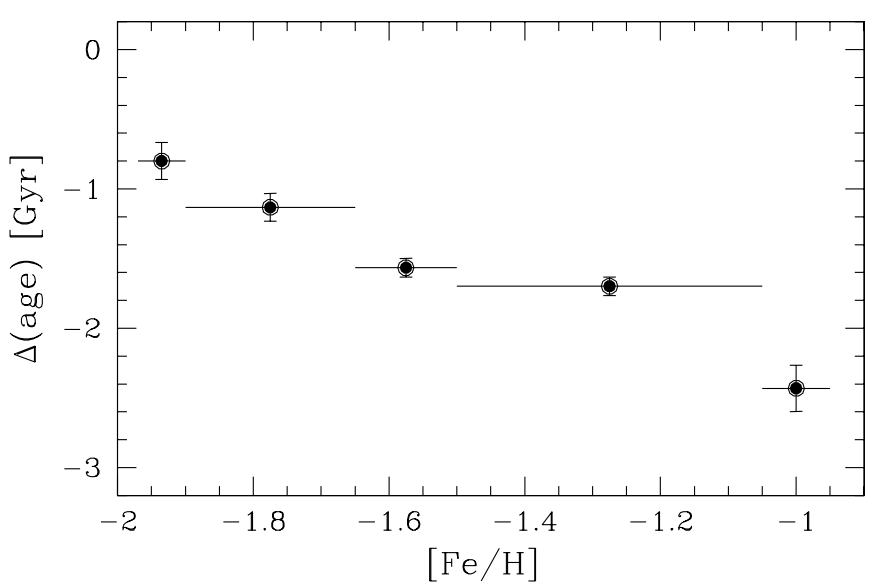

Figure 9. Age difference for each $\omega$ Cen sub-population, between the estimates based on $\mathrm{CNO}$-enhanced isochrones and on the actual $\mathrm{C}+\mathrm{N}+\mathrm{O}$ abundances, and the measurements provided by not $\mathrm{CNO}$-enhanced isochrones.

In the scenario in which star formation proceeds with a series of bursts, the first successive episode would have experienced an enrichment due only to the most massive supernovae from the first episode, and the following ones an enrichment due to a broader and broader range of supernova masses. Thus, a trend in $[\mathrm{O} / \mathrm{Fe}]$ with $[\mathrm{Fe} / \mathrm{H}]$ could be explained if the ratio of oxygen-to-iron yields from core collapse supernovae were a decreasing function of stellar mass, i.e., if low-mass supernovae were to make more oxygen relative to iron, compared to highmass supernovae.

Theoretical yields of oxygen and iron as a function of the initial mass of the supernova progenitor formally do not support this possibility (see, e.g., Woosley \& Weaver 1995; Limongi \& Chieffi 2006; Kobayashi et al. 2006), but are sufficiently uncertain to leave this option a priori relatively viable. The main uncertainty comes from the iron yield as a function of stellar mass being poorly constrained by supernova models, mainly due to the difficulty in locating the mass cut between ejecta and compact remnant that typically lies just within the iron layer of the pre-supernova structure. Nevertheless, this scheme would require lower mass stars to dominate the oxygen production, whereas all supernova models agree in predicting an increase of the oxygen yield with stellar mass, at least up to $\sim 30 M_{\odot}$, and in most models even beyond.

To explain the anomalous trend of $[\mathrm{O} / \mathrm{Fe}]$ with $[\mathrm{Fe} / \mathrm{H}]$ one may be tempted to appeal to the last resort of desperate situations, and invoke different IMFs for each successive episode of star formation, a very arbitrary and contrived scenario indeed. Moreover, note that this trend of increasing $[\mathrm{O} / \mathrm{Fe}]$ with increasing $[\mathrm{Fe} / \mathrm{H}]$ does not extend to the highest metallicity group in $\omega$ Cen, corresponding to the anomalous RGB-a and MS-a sequences in the CMD (cf. old ref.; Bellini et al. 2010), but here we have ascribed this whole metallicity group to the $p$-capture processed second generation.

In summary, even limiting ourselves to the normal, non- $p$-capture processed, first generation, we have to admit our failure in providing an explanation for the observed $[(\mathrm{C}+\mathrm{N}+\mathrm{O}) /$ $\mathrm{Fe}]$ and $[\mathrm{O} / \mathrm{Fe}]$ trends with $[\mathrm{O} / \mathrm{Fe}]$.

\section{CNO AND AGE OF $\omega$ CEN POPULATIONS}

The $\mathrm{C}+\mathrm{N}+\mathrm{O}$ abundance has a strong impact on the location of isochrones at the TO and SGB level, and therefore on age determinations. In order to estimate the effects 
Table 3

CNO Contents and Estimated Age Differences for the $\omega$ Cen $[\mathrm{Fe} / \mathrm{H}]$ Groups

\begin{tabular}{lccc}
\hline \hline$[\mathrm{Fe} / \mathrm{H}]$ & {$[(\mathrm{C}+\mathrm{N}+\mathrm{O}) / \mathrm{Fe}]$} & $\log \epsilon(\mathrm{C}+\mathrm{N}+\mathrm{O})$ & $\begin{array}{c}\Delta \text { age } \\
(\mathrm{Gyr})\end{array}$ \\
\hline$-1.97<[\mathrm{Fe} / \mathrm{H}]<-1.90$ & $0.24 \pm 0.04$ & $7.43 \pm 0.05$ & $-0.8 \pm 0.1$ \\
$-1.90<[\mathrm{Fe} / \mathrm{H}]<-1.65$ & $0.34 \pm 0.03$ & $7.69 \pm 0.04$ & $-1.1 \pm 0.1$ \\
$-1.65<[\mathrm{Fe} / \mathrm{H}]<-1.50$ & $0.47 \pm 0.02$ & $8.03 \pm 0.03$ & $-1.6 \pm 0.1$ \\
$-1.50<[\mathrm{Fe} / \mathrm{H}]<-1.05$ & $0.48 \pm 0.04$ & $8.24 \pm 0.05$ & $-1.6 \pm 0.1$ \\
$-1.05<[\mathrm{Fe} / \mathrm{H}]<-0.95$ & $0.81 \pm 0.12$ & $8.94 \pm 0.11$ & $-2.7 \pm 0.4$ \\
\hline
\end{tabular}

of the large variation of the overall CNO among $\omega$ Cen's stellar populations on the age measurement, we have calculated stellar models by including a variety of assumptions on the $\mathrm{C}+\mathrm{N}+\mathrm{O}$ abundance and spanning a metallicity range suitable for the $\omega$ Cen stellar populations. The adopted physical inputs and numerical assumptions are the same as in Pietrinferni et al. (2009), which presents evolutionary computations accounting for a $\mathrm{CNO}$ enhancement of a factor of $\sim 2$ relative to the "standard" $\alpha$-enhanced mixture. A fully consistent set of $\alpha$-enhanced isochrones with no $\mathrm{CNO}$ enhancement for various iron abundances (Pietrinferni et al. 2006), allows us to compare, in a fully homogeneous theoretical framework, CNO-enhanced and not-CNO-enhanced isochrones.

As expected, we find that, for a fixed TO and SGB brightness, the CNO-enhanced isochrones provide younger ages than isochrones corresponding to a canonical $\alpha$-enhanced mixture (Pietrinferni et al. 2006): as a rule-of-thumb we found the

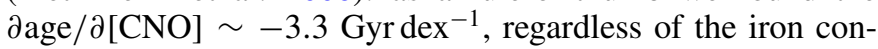
tent. By accounting for the observed $[\mathrm{CNO} / \mathrm{Fe}]$ abundance in $\omega$ Cen, we have estimated the difference between the age one would measure for each population by adopting appropriate CNO-enhanced isochrones and that obtained by using "normally" $\alpha$-enhanced isochrones, i.e., neglecting the measure CNO enhancement. The overall effect is to decrease the age estimate for the higher metallicity stars. The age decrease ranges from $\sim 0.5 \mathrm{Gyr}$ for stars with $[\mathrm{Fe} / \mathrm{H}] \sim-1.8$ up to $\sim 2 \mathrm{Gyr}$ for the most metal-rich stars with $[\mathrm{Fe} / \mathrm{H}] \sim-1.0$ (Figure 9), as listed in Table 3.

This result confirms that the measurement of relative ages of stellar populations in GCs requires accurate measurements of the overall $\mathrm{C}+\mathrm{N}+\mathrm{O}$ abundance. Indeed, it is well known that the double SGBs detected in NGC 1851, M22, and 47 Tucanae (Milone et al. 2008, Milone et al. 2011; Anderson et al. 2009; Piotto 2009) can be interpreted in quite different ways, depending on the CNO content of the single populations. In particular, the faint SGB can be associated either with a stellar population significantly older than the stars in the bright SGB (up to $1 \mathrm{Gyr}$ ), or to a second generation, almost coeval with the brighter SGB (age differences of 100-200 Myr), but with enhanced CNO (Cassisi et al. 2008; Ventura et al. 2009). This scenario has received further support from recent findings in M22 by Marino et al. (2009, 2011b): this GC hosts two stellar groups with different $s$-element and CNO abundances, and, in addition, the two groups of CNO-rich and CNO-poor stars also have different iron abundances, in close analogy with $\omega$ Cen.

In NGC 1851, results on possible internal variations of $\mathrm{C}+\mathrm{N}+\mathrm{O}$ are contradictory (Yong et al. 2009; Villanova et al. 2010). However, theoretical models, properly accounting for photometric signatures of the chemical peculiarities observed in the cluster sub-populations as provided by Sbordone et al. (2011), suggest that in NGC 1851 only a C+N+O-enhanced second generation can satisfy all the observational constraints given by CMDs in various photometric bands (Milone et al. 2008; Han et al. 2009).

Many studies have attempted to reconstruct the evolutionary history of $\omega$ Cen, by determining relative ages among the different stellar sub-groups, but none has been able to account for variations in the overall $\mathrm{C}+\mathrm{N}+\mathrm{O}$ abundance. Considering the complexity of its SGB morphology and of the metallicity distribution, and different assumptions regarding He enhancement among $\omega$ Cen populations, the relative ages for the various stellar populations as measured in different studies are quite different, ranging from a small or null age dispersion, as proposed by Sollima et al. (2005), to an age spread of the order of 2-3 Gyr (Hilker et al. 2004; Stanford et al. 2006) to 4 or more Gyr (Hughes \& Wallerstein 2000; Hilker \& Richtler 2000; Villanova et al. 2007).

It is beyond the scope of this paper to evaluate in detail how $\mathrm{C}+\mathrm{N}+\mathrm{O}$ abundances change the age dating obtained in the multiple cited works. However, we can identify some general trends. By using the same $\alpha$-enhanced isochrones adopted here, Villanova et al. (2007) found a large age difference ( $\sim 5$ Gyr) among the $\omega$ Cen populations. Specifically, they identify four groups of stars: (1) an old metal-poor group $([\mathrm{Fe} / \mathrm{H}] \sim-1.7),(2)$ an old population of metal-rich stars $([\mathrm{Fe} / \mathrm{H}] \sim-1.1),(3)$ a group of metal-intermediate young stars $([\mathrm{Fe} / \mathrm{H}] \sim-1.4)$, about 1-2 Gyr younger than the old components, and (4) a young metal-poor group $([\mathrm{Fe} / \mathrm{H}]$ $\sim-1.7$ ) up to $\sim 5$ Gyr younger than the old populations. When accounting for the $\mathrm{CNO}$ overabundance and using appropriate CNO-enhanced isochrones, the age estimate of the metal-rich component in Villanova et al. should be decreased by $\sim 2$ Gyr. As a consequence, the metal-rich population is no longer coeval with the most metal-poor population, but is slightly younger. Similarly, due to its enhanced CNO content, the intermediate metallicity $([\mathrm{Fe} / \mathrm{H}] \sim-1.4)$ population would be younger by $\sim 1$ Gyr relative to the estimates given by these authors. On the other hand, the age difference between the two groups of old and young metal-poor stars should not change as they have the same iron and $\mathrm{CNO}$ content.

Hilker et al. (2004) and Stanford et al. (2006) suggest that $\omega$ Cen has experienced a prolonged star formation of $\sim 2-4 \mathrm{Gyr}$ but, at odds with Villanova et al. (2007), with the most metalrich stars being younger. Similar conclusions have been reached by Hughes \& Wallerstein (2000) and Hilker \& Richtler (2000), who estimated that the more metal-rich stars were younger than the metal-poor ones by $\geqslant 3 \mathrm{Gyr}$, and 3-6 Gyr, respectively. In the case of Hilker et al. (2004) and Stanford et al. (2006), we predict that $\mathrm{CNO}$ effects on the relative-age estimate could increase the duration of the star formation in $\omega$ Cen up to $~ 4-6$ Gyr.

Sollima et al. (2005) suggest that the global star formation history of $\omega$ Cen should be confined to within $\sim 2 \mathrm{Gyr}$, with the metal-poor population $([\mathrm{Fe} / \mathrm{H}] \sim-1.7)$ representing the first and the major star formation burst. Our results suggest that the ages of the metal-intermediate and metal-rich stars given by these authors could be overestimated by $\sim 0.6$ and $\sim 1.5 \mathrm{Gyr}$, with respect to the metal-poor population. In any case, given their uncertainties of $2 \mathrm{Gyr}$, these corrections do not change the main result of their work, e.g., the suggestion that $\omega$ Cen stellar populations formed in a short time period.

As mentioned above, the goal of this paper is not to do a detailed analysis of previous age determinations. As discussed before there are contradicting results in recent literature. Our aim is simply to show that there is significant variation in the 
$\mathrm{C}+\mathrm{N}+\mathrm{O}$ content among $\omega$ Cen's populations that may easily change these datations. In addition to that caveat, it is worth noting that $\mathrm{He}$ enhancement is likely present in the O-poor, metal-rich stars. This occurrence should be taken into account when measuring the relative ages via isochrone fitting among the various sub-populations, since He affects the SGB shape (but not the luminosity level).

\section{CONCLUSION}

In this paper, we have presented $\mathrm{C}, \mathrm{N}, \mathrm{O}$ abundances for $77 \omega$ Cen RGB stars in the metallicity range $\sim-2.0<$ $[\mathrm{Fe} / \mathrm{H}]<-0.9$. We have coupled our results with the ones of our previous work on $\mathrm{Na}-\mathrm{O}-\mathrm{La}$ abundances presented in Marino et al. (2011a). From our analysis we found:

1. a correlation between the total CNO and the iron abundance, with the most metal-rich population being enhanced by $\sim 0.5$ dex in $[(\mathrm{C}+\mathrm{N}+\mathrm{O}) / \mathrm{Fe}]$ relative to the most metal-poor one;

2. $\mathrm{O}$ and $\mathrm{C}$ grows with $[\mathrm{Fe} / \mathrm{H}]$ and $[\mathrm{La} / \mathrm{Fe}]$ in the O-rich/Na-poor stars;

3. stars selected on the basis of their position on the $\mathrm{Na}-\mathrm{O}$ plane, show defined chemical patterns in their light elements $\mathrm{C}, \mathrm{N}, \mathrm{O}$, and $\mathrm{Na}$ as a function $\mathrm{CNO}, \mathrm{Fe}$, and $\mathrm{La}$, that allow us to distinguish between a first and a second generation of stars, each possibly resulting from a series of separate bursts of star formation; and

4. $[\mathrm{La} / \mathrm{Fe}]$ correlates tightly with $[\mathrm{Fe} / \mathrm{H}]$, following almost precisely the same trend regardless of the $\mathrm{O} / \mathrm{Na}$ abundances.

In an attempt to make sense of these observational trends we have explored two (speculative) scenarios for the formation and evolution of this most puzzling object. In one option the system has evolved monolithically, i.e., remaining chemically homogeneous at each time. Thus, within less than $\sim 30-40 \mathrm{Myr}$ a series of bursts of star formation each enriched in iron and $\alpha$ elements by core-collapse supernovae from the previous burst(s) would have been followed each by a secondary burst of star formation originating from material ejected by AGB stars, heavily processed by $p$-captures. The formation of the whole stellar population inhabiting the cluster today would have taken less than $\sim 2 \times 10^{8}$ yr. Alternatively, rather than being sequential in time, such primary and secondary bursts of star formation would have taken place separately in space, before their products could merge at the bottom of the potential well. In both cases, secondary bursts would be fed via cooling flows made of AGB ejecta, leading to more centrally concentrated second generations, which indeed appears to be so in this (and other) clusters (Sollima et al. 2005; Bellini et al. 2009; Johnson \& Pilachowski 2010). We admit that both scenarios require a series of very contrived and ad hoc assumptions, yet the extraordinary complexity of $\omega$ Cen may not admit simple solutions.

The CNO abundance affects the determination of the relative ages of cluster subpopulations via isochrone fitting of the TO SGB region. In light of our results, we have discussed this issue in the case of $\omega$ Cen by comparing isochrones with standard and with enhanced CNO, with the latter ones giving younger ages for the same TO luminosity. Although the determination of relative ages of the $\omega$ Cen sub-populations is beyond the aims of our study, we argue that a trend in $\mathrm{CNO} / \mathrm{Fe}$ can help reducing large age spread among the various subpopulations, as found by some studies in the literature.
We thank the anonymous referee whose suggestions have significantly improved this work. A.P.M., G.P., S.C. and A.A. are founded by the Ministry of Science and Technology of the Kingdom of Spain (grant AYA 2010-16717). A.P.M. and A.A. are also founded by the Instituto de Astrofísica de Canarias (grant P3-94).

\section{REFERENCES}

Anderson, A. J. 1998, PhD thesis, Univ. California, Berkeley

Anderson, J., Piotto, G., King, I. R., Bedin, L. R., \& Guhathakurta, P. 2009, ApJ, 697, L58

Bedin, L. R., Piotto, G., Anderson, J., et al. 2004, ApJ, 605, L125

Bekki, K., \& Freeman, K. C. 2003, MNRAS, 346, L11

Bekki, K., \& Norris, J. E. 2006, ApJ, 637, L109

Bellini, A., Bedin, L. R., Piotto, G., et al. 2010, AJ, 140, 631

Bellini, A., Piotto, G., Bedin, L. R., et al. 2009, A\&A, 507, 1393

Bensby, T., Feltzing, S., \& Lundström, I. 2004, A\&A, 415, 155

Brown, J. A., \& Wallerstein, G. 1993, AJ, 106, 133

Busso, M., Gallino, R., \& Wasserburg, G. J. 1999, ARA\&A, 37, 239

Calamida, A., Bono, G., Stetson, P. B., et al. 2009, ApJ, 706, 1277

Carretta, E., Gratton, R. G., Lucatello, S., Bragaglia, A., \& Bonifacio, P. 2005, A\&A, 433, 597

Cassisi, S., Salaris, M., Anderson, J., et al. 2009, ApJ, 702, 1530

Cassisi, S., Salaris, M., Pietrinferni, A., et al. 2008, ApJ, 672, L115

Castelli, F., \& Kurucz, R. L. 2004, in IAU Symp. 210, Modelling of Stellar Atmospheres, ed. N. Piskunov et al. (Cambridge: Cambridge Univ. Press), arXiv:astro-ph/0405087

D’Antona, F., Caloi, V., \& Ventura, P. 2010, MNRAS, 405, 2295

D'Antona, F., D'Ercole, A., Marino, A. F., et al. 2011, ApJ, 736, 5

D’Antona, F., Stetson, P. B., Ventura, P., et al. 2009, MNRAS, 399, L151

D'Ercole, A., D’Antona, F., Ventura, P., Vesperini, E., \& McMillan, S. L. W. 2010, MNRAS, 407, 854

D'Ercole, A., D’Antona, F., \& Vesperini, E. 2011, MNRAS, 415, 1304

Dupree, A. K., Strader, J., \& Smith, G. H. 2011, ApJ, 728, 155

Han, S.-I., Lee, Y.-W., Joo, S.-J., et al. 2009, ApJ, 707, L190

Hilker, M., Kayser, A., Richtler, T., \& Willemsen, P. 2004, A\&A, 422, L9

Hilker, M., \& Richtler, T. 2000, A\&A, 362, 895

Hill, V., Plez, B., Cayrel, R., et al. 2002, A\&A, 387, 560

Hughes, J., \& Wallerstein, G. 2000, AJ, 119, 1225

Iben, I., Jr., \& Renzini, A. 1983, ARA\&A, 21, 271

Ivans, I. I., Sneden, C., Kraft, R. P., et al. 1999, AJ, 118, 1273

Johnson, C. I., \& Pilachowski, C. A. 2010, ApJ, 722, 1373

Kobayashi, C., Umeda, H., Nomoto, K., Tominaga, N., \& Ohkubo, T. 2006, ApJ, 653,1145

Lee, Y.-W., Joo, J.-M., Sohn, Y.-J., et al. 1999, Nature, 402, 55

Lee, Y.-W., Joo, S.-J., Han, S.-I., et al. 2005, ApJ, 621, L57

Limongi, M., \& Chieffi, A. 2006, ApJ, 647, 483

Marino, A. F., Milone, A. P., Piotto, G., et al. 2009, A\&A, 505, 1099

Marino, A. F., Villanova, S., Piotto, G., et al. 2008, A\&A, 490, 625

Marino, A. F., Milone, A. P., Piotto, G., et al. 2011a, ApJ, 731, 64

Marino, A. F., Sneden, C., Kraft, R. P., et al. 2011b, A\&A, 532, A8

Matteucci, F., \& Greggio, L. 1986, A\&A, 154, 279

Milone, A. P., Bedin, L. R., Piotto, G., et al. 2008, ApJ, 673, 241

Milone, A. P., Piotto, G., Bedin, L. R., et al. 2011, arXiv:1109.0900

Norris, J. E. 2004, ApJ, 612, L25

Norris, J. E., \& Da Costa, G. S. 1995, ApJ, 447, 680

Norris, J. E., Freeman, K. C., \& Mighell, K. J. 1996, ApJ, 462, 241

Pancino, E., Ferraro, F. R., Bellazzini, M., Piotto, G., \& Zoccali, M. 2000, ApJ, 534, L83

Pietrinferni, A., Cassisi, S., Salaris, M., \& Castelli, F. 2006, ApJ, 642, 797

Pietrinferni, A., Cassisi, S., Salaris, M., Percival, S., \& Ferguson, J. W. 2009, ApJ, 697, 275

Piotto, G. 2009, in IAU Symp. 258, The Ages of Stars, ed. E. E. Mamajek, D. R. Soderblom, \& R. F. G. Wyse (Cambridge: Cambridge Univ. Press), 233

Piotto, G., Villanova, S., Bedin, L. R., et al. 2005, ApJ, 621, 777

Raiteri, C. M., Gallino, R., Busso, M., Neuberger, D., \& Kaeppeler, F. 1993, ApJ, 419, 207

Renzini, A. 2008, MNRAS, 391, 354

Sbordone, L., Salaris, M., Weiss, A., \& Cassisi, S. 2011, A\&A, 534, A9

Simmerer, J., Sneden, C., Cowan, J. J., et al. 2004, ApJ, 617, 1091

Smith, V. V., Suntzeff, N. B., Cunha, K., et al. 2000, AJ, 119, 1239

Sollima, A., Pancino, E., Ferraro, F. R., et al. 2005, ApJ, 634, 332

Sneden, C. A. 1973, PhD thesis, Univ. Texas, Austin 
Stanford, L. M., Da Costa, G. S., \& Norris, J. E. 2010, ApJ, 714, 1001

Stanford, L. M., Da Costa, G. S., Norris, J. E., \& Cannon, R. D. 2006, ApJ, 647, 1075

Suntzeff, N. B., \& Kraft, R. P. 1996, AJ, 111, 1913

Valcarce, A. A. R., \& Catelan, M. 2011, A\&A, 533, A120

Vanbeveren, D., Mennekens, N., \& De Greve, J. P. 2011, arXiv:1109.2713

Ventura, P., Caloi, V., D’Antona, F., et al. 2009, MNRAS, 399, 934
Ventura, P., \& D'Antona, F. 2009, A\&A, 499, 835

Villanova, S., Geisler, D., \& Piotto, G. 2010, ApJ, 722, L18

Villanova, S., Piotto, G., King, I. R., et al. 2007, ApJ, 663, 296

Wheeler, J. C., Sneden, C., \& Truran, J. W., Jr. 1989, ARA\&A, 27, 279

Woosley, S. E., \& Weaver, T. A. 1995, ApJS, 101, 181

Yong, D., Grundahl, F., D’Antona, F., et al. 2009, ApJ, 695, L62

Zoccali, M., Lecureur, A., Barbuy, B., et al. 2006, A\&A, 457, L1 